\title{
Effects of Phosalone plant pesticide on male fertility by using experiment on Rat
}

\author{
Professor Dr. Sorush Niknamian \\ Board Member of Weston A Price Foundation, Washington DC, USA \\ Email: so.niknamian@gmail.com
}

Infertility is a major problem for couples and half of the total number of infertilities belong to men, diagnosable in $40 \%$ and pathologically undiagnosable in $60 \%$ of the cases. Complications of exposure to pesticides is a concern, with a considerable share in male infertility. Phosalone is an organophosphate insecticide usually used to control various types of pests in agriculture. Considering the extensive use of this compound worldwide, the present study examined the effects of environmental agents related to organophosphate on male infertility. To perform the experiments, 16 adult male rats were randomly assigned to four groups with four each. Group 1 received routine rat feed and water as the first control group; Group 2 received low doses (60 mg/kg); Group 3 medium doses ( $90 \mathrm{mg} / \mathrm{kg}$ ); and Group 4 high doses of Phosalone (120 mg/kg). After 48 days (end of the treatment), the rats were weighed and anesthetized using ether. Then, their tail of epididymis was removed, and sperm parameters, including number, motility, and viability, were evaluated and compared. Moreover, their reproductive organs were removed and the sexual tissue was processed and underwent histopathological examinations.

In addition, in this study, important biochemical parameters in infertility such as the level of sex hormones ( $\mathrm{LH}, \mathrm{FSH}$, and testosterone) were evaluated using the ELISA test. Results showed that Phosalone decreased sperm motility, viability, and number in a dose-dependent manner. The level of FSH and LH was increased, and that of testosterone was reduced. This increase in FSH and LH must have occurred as the result of reduced testosterone, and all these changes were dependent on the dose of Phosalone. Results of histological studies also revealed that the trend of degeneration in the tissues of testes, epididymis, and seminal vesicle was completely dose-dependent. Phosalone has considerable effects on reproductive indices and can cause serious damage and impose undesirable effects on the number and motility of sperms, causing infertility by altering the concentration of hormones 
Keywords: Pesticides: Male infertility: Organophosphate: Phosalone: Sex hormones. 


\section{Introduction}

Recent advance in industries and biotechnology has led to products improving the conditions of living for humans. However, along with these advances, a large number of physical, chemical, and biotechnical agents have been created which affect the environment. Based on the sensitivity of reproduction, these agents have caused considerable damage to the female and male reproductive system. In the past 50 years, human exposure to environmental and occupational chemicals has considerably increased, and pesticides have the greatest share among these chemicals (Toppari et al, 1996). Complications of exposure to pesticides for male fertility is a concern and exposure to pesticides, especially by farmers, is a major share in male infertility (Colborn et al, 1993, Wissem et al, 2011). The negative effects of pesticides on fertility is minimally known. In general, however, it can be claimed that toxicity caused by pesticides occurs through several direct and indirect biological mechanisms (Bretveld et al, 2006, Hanke \& Jurewicz, 2004). Pesticides infiltrate the blood circulation and directly lead to the production of infertile sperms by damaging and destroying the cells of the testes tissue or as mutagenic substances in cells (Larsen et al, 1998). Some pesticides are agents disrupting the endocrine glands, causing hormonal imbalance and disruptions in male fertility. The synthesis, storage, release, and bonding of hormones are affected by them, leading to a change in the normal level of reproductive hormones (Souheila et al, 2011, Tabb \& Blumberg, 2006). They disrupt the normal functioning of reproductive hormones and indirectly decrease sperm production or production of sperms with abnormal morphology by disturbing hormonal paths in cell differentiation and spermatogenesis (Whorton, 1994, Melissa, 2008). Other negative effects of pesticides in male infertility include a disorder in the normal differentiation of chromosomes during meiosis by causing number anomalies in sperm chromosomes (Wasim et al, 2009).

A study by Kenivald et al (2000) showed that atrazine pesticide reduces the number of epididymis sperms and sperm motility. They also showed that Lidig cells are observed in the testes' interstitial tissue irregularly. Najai et al. (2013) reported that atrazine in high doses can decrease the number and progressive motility of sperms. In addition, the number of dead or immotile sperms is increased.

Kanokporn et al. (2014) examined the safety of biological insecticides produced from some plants on the reproductive organ of male rats. Rats were treated with the oral doses of 2, 10 , and $50 \mathrm{mg} / \mathrm{kg}$ of a biological insecticide for 60 consecutive days, versus a single dose of 2 $\mathrm{mg} / \mathrm{kg}$ of a carbamate insecticide, and then compared. Authors observed that the weight of testes, prostate, and seminal vesicle was significantly reduced in the rats treated with biological and carbamate pesticide compared to the control group. Nevertheless, reduction 
in the density of sperms and histopathological changes in the testes were only observed in the rats treated with carbamate and not in those treated with biological insecticides.

Nithya et al. (2015) induced reproductive toxicity using lindane pesticide and examined the level of male sex hormones, antioxidants, and histopathology of testes in rats. In this study, 10 healthy rats were treated with $5 \mathrm{mg} / \mathrm{kg}$ of lindane for 30 days. The level of male hormones and antioxidants was reduced in the group receiving lindane compared to the control group. Histopathological examination of the rats' testes showed fission in seminiferous tubules, separation of germ cells from the base membrane, and abnormal intracellular space between seminiferous tubules.

Xia Geng et al. (2015) treated a number of adult rats using gavage with the dose of 0, 33, 54, $75,108 \mathrm{mg} / \mathrm{kg}$ over 60 days using Malathion pesticide, and evaluated histological, biochemical, and serological alterations. Malathion decreased sperm number and motility, body and testicular weight, and mild and severe alterations in seminiferous tubules in rats. They observed that Malathion at the dose of $54 \mathrm{mg} / \mathrm{kg}$ increased the level of apoptosis in sperm cells, also significantly changing the activity of testicular enzymes through reducing reproductive hormones, e.g. FSH and LH. These results indicated that Malathion can have destructive effects on rats' reproductive system.

Bhawana et al. (2016) conducted a study to investigate whether dichloro-dimethyl phosphate had diagnosable undesirable effects on the testes of male rats. Animals were treated with oral dichloro-dimethyl phosphate at the dose of $20 \mathrm{mg} / \mathrm{kg}$ for 45 days. Their body and sex organ weight were significantly decreased. Also, a significant reduction was observed in sperm density along with $80 \%$ infertility in the treated animals.

This substance disrupted the normal functioning of hormones and indirectly decreased sperm production or production of sperms with abnormal morphology by disturbing hormonal paths in cell differentiation and spermatogenesis (Whorton, 1994, Melissa, 2008). Other negative effects of pesticides in male infertility include a disorder in the normal differentiation of chromosomes during meiosis by causing number anomalies in sperm chromosomes (Wasim et al, 2009).

\section{Materials and Methods}

In this study, 16 adult male rats (8-12 weeks of age) with the mean weight of $250 \pm 1.2 \mathrm{~g}$ were selected, randomly assigned to four groups of four, and received phosalone orally through gavage feeding for 48 days. Group 1 was the control group, including 4 male rats which received only distilled water and usual feed for 48 days. Group 2 comprised 4 male rats which, in addition to usual feed, received $60 \mathrm{mg}$ of phosalone (low dose) orally through gavage. Group 3 included male rats which, in addition to the usual feed, received $90 \mathrm{mg}$ of 
phosalone (medium dose). Finally, Group 4 consisted of rats which, in addition to the usual feed, received $120 \mathrm{mg}$ (high dose) of phosalone through gavage.

Animals were kept in standard conditions, with the optimal temperature of $22-24^{\circ} \mathrm{C}$, and 12 hours of light in clean cages. They were constantly fed on special rat feed and water.

After 48 days equal to a period of spermatogenesis in rats, the rats in all groups were anesthetized using ether and their left testicle was removed by cutting the scrotum. Then, the tail of epididymis was separated by scissors and transferred to $1000 \mu \mathrm{l}$ of the Ham.sf10 culture medium inside a central Petri dish. To remove the sperms from the tail of epididymis in the removed tissue, some cuts were gently created using an insulin syringe. Petri dishes containing the prepared suspension were kept in the incubator for $30 \mathrm{~min}$ at $37^{\circ} \mathrm{C}$ and $\mathrm{CO} 2$ pressure of $5 \%$. Then, the samples were removed from the incubator, and the experiments were performed on them. Eventually, the final experiments regarding the parameters of interest (sperm-related parameters, biochemical and histopathological tests, and important biochemical parameters in infertility) were performed for each animal in the form of developed questionnaires and, then, evaluated.

\section{Analysis of sperm number and motility}

To determine the level of motility of sperms, the standard Makler Chamber method was applied. This instrument has applications for enumerating sperms. Also, due to its special structural properties, it is widely used for evaluating sperm motility. This tool has a main part and a metal base. The center of the main part includes a broad disk where the sample must be placed. Around this glass disc, there are four quartz columns standing $10 \mu \mathrm{m}$ above the level of the disk. Also, a glass lid is placed on the disc. At the center of the disc, there is a plate with the area of $10 \mathrm{~mm}^{2}$ which is divided into 100 squares (10x10) for enumerating sperms. If the sperms in one row are enumerated and multiplied by $10^{6}$, the number of sperms in each $\mathrm{mm}^{3}$ of the sample is obtained. As a space with the height of $10 \mu \mathrm{m}$ exists between the disc and the lid, the sperms easily swim in the medium and their motility can be better examined (Figure 1).

Fig.1.

To examine sperm number and motility, $10 \mu \mathrm{l}$ of each sample was placed in the center of the Makler chamber. After placing the lid, the samples were examined with a light microscope at the magnification of $\mathrm{x} 200$. To this end, on each slide, 100 sperms were counted, and sperm number and motility were determined based on World Health Organization's guidelines (Farag et al, 2000). Based on these guidelines, the sperms were divided into four groups: immotile, motile with fast progressive motility, motile with slow progressive motility, and motile with non-progressive motility. 
Evaluation of viability is performed with different staining tests. Here, the Eosin-Nigrosin Staining was performed. To perform this test, $10 \mu \mathrm{L}$ of each sample was taken by a sampler and smeared on a clean slide. Then, $10 \mu \mathrm{L}$ of the eosin-nigrosin solution was added to it. With pipetting, the sample and dye were mixed and covered with a cover slip, and viewed under a light microscope at the magnification of x100. Finally, by counting 100 sperms and determining the number of sperms not stained (viable) and red sperms (dead), the viability of each sample was evaluated (Figure 2).

Fig.2.

\section{Hormone measurement}

A. Taking blood samples from animals: After 48 days of treatment with phosalone and anesthetizing the groups, the blood samples were taken from the rats' hearts. Then, the blood in the syringe containing heparin (anticoagulant) was quickly transferred to small tubes. Afterwards, the blood-containing tubes were placed in the centrifuge device for $10 \mathrm{~min}$ at $5000 \mathrm{rpm}$. Next, the tubes were removed from the device, blood serum which had formed a separate phase on the coagulated part was carefully removed by a sampler, poured into normal tubes, and kept at $-70^{\circ} \mathrm{C}$ until hormone measurement.

B. Hormone measurement using the ELISA method: To evaluate the level of sex hormones including FSH and LH, the ELISA technique was applied. An ELISA reader (also known as microplate reader and photometric microplate reader) is a specialized spectrophotometer for reading the results of the ELISA test. This device is utilized for determining the presence of antibodies or specialized antigens in samples. This technique is based on the identification of an antigen or antibodies on a solid surface in a direct or secondary manner with the aid of labeling antibodies and production of products which can be read via the spectrophotometer.

\section{Results and Discussion}

Weight variations of body, testicle, and epididymis

Examination of weight of the groups showed that, in the control group and the group receiving a low dose $(60 \mathrm{mg} / \mathrm{kg})$, testes and epididymis had a normal appearance, whereas they looked smaller in the groups treated with medium $(90 \mathrm{mg} / \mathrm{kg}$ ) and high doses $(120 \mathrm{mg} / \mathrm{kg})$ compared to the control group. In the groups treated with phosalone, by increasing the dose, body, testicle, and epididymis weight were reduced; this reduction was considerable in the group receiving the high dose compared to the control group. Results showed a significant 
difference $(\mathrm{p}<0.05)$ existed between the groups in terms of body, testicle, and epididymis weight. Supplementary data are presented in Table 1.

Table1.

Density, motility, and viability of sperms Results of examining sperm density and viability in the tail of epididymis showed that these factors were significantly reduced in a dosedependent manner in the groups receiving phosalone compared to the control group. These differences were significant $(\mathrm{p}<0.05)$ across the four groups. In addition, results indicated that the progressive motility of the sperms was decreased upon increasing the dose of phosalone, whereas the slow or non-progressive motility and the number of immotile sperms were considerably increased. Details are given in Table 2.

Table2.

Using the eosin-nigrosine staining method, the numbers of dead (pale violet) and viable (nonstained) sperms were counted by a light microscope in each sample to evaluate sperm viability. As shown in Table 2, the number of viable sperms was decreased and that of the dead sperms was remarkably increased in a dose-dependent manner in the rats receiving phosalone. The increase in the number of dead sperms in the groups treated with 90 and 120 $\mathrm{mg} / \mathrm{kg}$ of phosalone (medium and high doses, respectively) was considerable compared to the control group. These differences were significant at the level of $p<0.05$ across the four groups (Figure 3).

Table3.

\section{Hormone measurement}

It was shown that the level of serum testosterone was significantly reduced in the groups receiving phosalone compared to the control group in a dose-dependent manner. LH and FSH were also measured in the blood serum of all the groups. The level of these hormones was increased in the groups treated with phosalone compared to the control group, but these differences were not significant $(\mathrm{p}>0.05)$ (Table 3).

\section{Discussion:}

About $20 \%$ of young couples suffer from infertility and $50 \%$ of the causes of infertility are related to men. Various factors contribute to male infertility which, in most cases, have to do with sperm quality. Complications of exposure to pesticides for male fertility is a concern and pesticides have a major share in male infertility, suppressing the reproductive performance and sexual behavior of humans and laboratory animals (Kidd et al, 2001). Findings of the present study revealed that the oral consumptions of phosalone over 48 days decreased body, testicle, and epididymis weight in the treated groups compared to the control group. Body weight of the treated animals was significantly less than that of the control group. Other 
studies have reported similar findings. Wissem et al. reported that the body weight of animals receiving $250 \mathrm{mg} / \mathrm{kg}$ of an organophosphate was decreased, which was confirmed the present study as well (Schlegel et al, 1991). Based on studies worldwide, it is predicted that growing children, especially infants and fetus, should be more sensitive to the toxicity of organophosphates. Researchers in studies to support this hypothesis showe that the weight of the fetus is reduced in women exposed to the organophosphates chlorpyrifos (Wissem et al, 2011).

Ambadi et al. (2011) reported that weight reduction following the prescription of organophosphates chlorpyrifos was improved with treatment via vitamin $\mathrm{E}$. This finding showed that oxidative stress might be involved in suppressing body weight affected by chlorpyrifos and the results of the present study also showed weight reduction in rats. Still, a study by Joshi et al. indicated that body weight did not change as the result of the effect of organophosphates. On the contrary, another research reported that body weight increased via exposure to a type of organophosphates, which was due to the increased fat tissue (Ambali et al, 2011). Numerous studies have reported that many pesticides affect the pituitary, hypothalamus or both, thus reducing the weight of body organs. In our study, the decrease in epididymis and testicle weight might be due to the unavailability of sufficient amounts of male hormones or the anti-androgenic activity of phosalone. Another reason for the decreased weight of testes can be the reduction in the number of various classes of spermatogenic cells and the diameter of seminiferous tubules. This reduction can also be due to the reduction in the population in seminiferous tubules of testes or interstitial cells of seminiferous tubules (Joshi et al, 2007). It seems that by stopping mitosis divisions and inducing cell death, toxins decrease germinal cells, finally leading to a reduction in testes weight. Epididium weight is mostly dependent on the mass of various spermatogenic cells and a decrease in its weight may be due to the reduction in the volume of tubules as well as number of germinal cells and long spermatids. Moreover, stopping spermatogenesis and inhibiting steroid biosynthesis of leydig cells might contribute to the reduction in epididymis weight (Joshi \& Sharma, 2011). Body, testicle, and epididymis weight showed a significant reduction in treatment versus control groups. This finding confirmed the effectiveness of complications of this pesticide. The use of different doses of phosalone in this study decreased some parameters in testes. Examinations showed that organophosphate toxins reacted with cell macromolecules and, by increasing peroxidation, induced cell death. It could also postpone mitosis or stop it (Joshi et al, 2007). Organophosphate toxins alter the activity of antioxidant enzymes which induce reproductive damages, probably by creating free radicals and reactive oxygen species. The phosphorous organic compounds react with the main cell macromolecules such as proteins, nucleic acids, and lipid. Organophosphate causes atrophy in leydig and Sertoli cells, decreasing the level of blood serum testosterone 
(Jasuja et al, 2013). Research shows that this type of toxins in the long-term decreases spermatogenic, leydig cells, and testosterone, and increases LH and FSH (Taib et al, 2014). Results of the present study showed the same effects on rats' testicle tissue and cells treated with various doses of phosalone compared to the control group over 48 days. Based on our studies and those by others, it can be concluded that the effects of these toxins depend on their dosage and duration of exposure to them. It seems that phosalone decreases germinal cells by stopping mitosis and inducing cell death. These cells are essential for spermatogenesis, and reduction in them affects cell classes such as spermatocytes, thus leading to a reduction in the number of sperms (Joshi et al, 2007). As mean level of testosterone shows a considerable dose-dependent reduction in the treatment groups, after the reduction of ley

dig cells responsible for secreting testosterone in males, it is expected that the level of this hormone should be reduced in blood serum. Various studies represent that organophosphates reduce the level of serum steroid hormones by increasing catabolism, directly affecting testes tissue, and directly and indirectly affecting the endocrine system (Fattahi et al, 2009). Numerous factors contribute to the reduction of testosterone. By inhibiting steroids, a defect in the production of androgens, or degeneration of leydig cells, the level of testosterone is reduced (Taib et al, 2014). However, based on our results, the decrease in the level of testosterone has a direct association with the reduction in the number of leydig cells. In this study, the serum level of LH and SFH was increased, but this increase was not significant. Organophosphates increase LH and FSH by preventing the inhibition of return. It seems that phosalone, which has increased these two hormones in this study, belongs to the same group and increases LH and FSH by preventing the inhibition of return. LH plays a significant role in starting and continuing destruction in different stages of spermatogenesis and its increase can reduce testes tissue cells. For the production, maturation, and transfer of sperms, the existence of appropriate levels of sex hormones is essential. A change in the level of testosterone, LH, and FSH can disrupt the process of sperm production (Farag et al, 2010). Hormonal changes can have various reasons; they may be central or peripheral, i.e. sometimes hormonal changes may be due to the direct damage to pituitary and destruction of the secretion of hormones from the pituitary and hypothalamus. This brain damage may occur in the case of phosalone, although we did not examine the histopathology of brain tissue. Another reason for hormone secretion disruption is the direct tissue damage. This includes reduction in or damage to leydig cells which secrete testosterone under the effect of LH. Yet, another reason for hormonal changes may be a disorder in hormone metabolism. As LH and FSH are protein hormones with hepatic and renal metabolism, renal and hepatic damage may have caused a disorder. However, testosterone is a lipid hormone with a mostly hepatic metabolism. Therefore, the 
reason may be the toxicity of hepatic cells. Sujatha et al. (2001) evaluated the effects of the survival of agriculture toxins on the level of health and blood levels of farmers. They found that long-term effects of toxins destroy the tissue of the liver, kidneys, and testes, and leads to a considerable decrease in testosterone level (Adamkovicova et al, 2016). Another reason for $\mathrm{LH}$ increase is that the toxic inhibits the negative feedback path of $\mathrm{LH}$ and, by preventing the inhibition of return, increases this hormone. On the other hand, reduction of testosterone increases LH. Simultaneously with the increase in LH, FSH is increased. Epididymis plays an important role in male fertility by providing an optimal liquid medium for the maturation and storage of sperms. The liquid secreted by epididymis is regulated by neurotransmitters which are paracrine and endocrine hormones. The physiologic and biochemical integrity of epididymis also depends on the shortage of androgens. Thus, based on the results of the present study, the shortage of androgens may have caused a marked reduction in the diameter of tubules, density of sperm in the tail of epididymis, and a change in its plasma composition (Joshi \& Sharma, 2011). Some studies have shown that, in summer, the level of sperm is decreased among farmers compared to winter, and the reason has been attributed to the use of toxins in summer in a large amount (Sujatha et al, 2001).

Patrick et al. examined the exposure of organophosphate pesticides and reduction in sperm density, showing that the longer the exposure to this pesticide, the further the reduction in sperm density would be (Najafi et al, 2013). Variation in sperm parameters can be attributed to the direct effect on testicle tissue, leading to a disorder in reproductive function, e.g. reduction in density, motility, and morphology of sperms. Specifically, the disorder in strong bonds of Sertoli germ cells leads to a defect in spermatogenesis. Deep damage to testes demonstrates the destruction of seminiferous tubules and germinal cells, leading to a disorder in the initial growth of sperm. In addition, exposure to phosalone at low doses affects the functioning of steroid hormones involved in the regulation of fertility processes. Reduction in sperm density and quality is correlated with the reduction in the level of testosterone and oxidative damage, resulting from the inhibition of antioxidant enzyme activity. These findings show that exposure to phosalone leads to a disorder in the process of spermatogenesis along with a marked reduction in sperm quality. In general, oxidative damage plays a role in the genotoxic and fertility-related effects under the effect of various metals and organophosphorus pesticides, in line with the results presented here. There is evidence that exposure to arsenic and organophosphate toxins increases ROS in blood and tissues. Increased lipid peroxidation is associated with a change in sperm membrane, reduction in sperm motility, and decrease in fertility potential. Moreover, phosalone can alter the quality of sperm and integrity of sperm DNA and related proteins by producing free radicals in testes, all exacerbating testicle dysfunction. The excessive production of free radicals leads to the probable reduction of mitochondrion membrane along with the 
reduction in accessible energy which may prevent the motility of sperms (Jasuja et al, 2013). Sperm stimulation is affected by the enzymatic activities of oxidative phosphorylation. The abundance of adenosine triphosphate (ATP) is very important for the normal motility of sperms. Reduction of ATP reduces sperm motility, which may eventually lead to infertility. Reduction in the number of sperms in the tail of epididymis is an indicator for the reduction of spermatogenesis as the result of the toxicity of any agent. Biologically speaking, the secretion of gonadotropins is vital for the normal production, growth, and maturation of sperms by testes and epididymis. The decrease in the number of sperms may be due to the change in androgen gonadotropins. Epididymis sperms strongly depend on testosterone and epididymis proteins for their final maturation, development for progressive motility, and fertility capacity. Decrease in sperm motility in the tail of epididymis reflects the low ability of sperms for interaction with the plasma membrane of the ovum. Cyclic nucleotides, especially cyclic adenosine monophosphate (cAMP), are an internal regulator of sperm motility. In addition, it has been reported that sperms develop motility following passage from the epididymis, which may be related to the increase in intracellular cAMP. Phosalone may reduce adenylate cyclase in the epididymis. As a result, no cAMP is produced which eventually leads to the reduction in sperm motility (Joshi \& Sharma, 2011). The difference in the color of healthy and dead sperms as the result of eosin-nigrosin staining in this study indicates that the severity of anomalies in sperm parameters was higher in rats exposed to higher doses. Furthermore, the formation of free radicals can decrease sperm quality. Free radicals can affect the activity of mitochondrion enzymes, leading to a reduction in cell's ability for maintaining the level of their ATP and disorder in the structure of sperm microtubules. Thus, in this way, they disrupt their normal functioning. Slight deprivation of ATP may reduce sperm motility. This mechanism of defect interprets the quality of sperms by decreasing sperm density and motility, and increasing the number of death sperms in the groups treated with phosalone. Taib et al. reported that the prescription of Fenitrothion at a low dose in the diet can lead to a slight decrease in the concentration and motility of sperms and morphological changes in them (Taib et al, 2014). On the other hand, they indicated that Dichlorvos leads to the reduction of the sperm motility percentage without causing any change in the sperm or its morphology. This may be due to the inability of Dichlorvos in maintaining ATP synthesis in sperm mitochondrion (Patrick et al, 2016). Moreover, exposure to environmental toxins including organophosphates can cause oxidate stress which inhibits the activity of antioxidant enzymes and increases lipid peroxidation in the sperm. Organophosphorus pesticides can damage sperm DNA through oxidative stress mechanism. Seminal vesicles play a major role in male fertility, and its secretions are necessary for the normal functioning of sperms. Seminal vesicle is an accessory gland of the reproductive organ, producing secretions which are essential for male reproductive 
functioning. It consists of two coiled tubes. When this organ is sectioned, the same tubes in various directions can be seen inside. These tubes have a folded mucus covered by pseudo stratified columnar epithelium. Its lamina propria is rich in elastic fibers, surrounded by a thin layer of smooth muscle (Wissem et al, 2011). In this study, phosalone leads to degenerative changes in seminal epithelium in addition to the absence of sperm in the lumen in treatment groups compared to the control group. As sexual differentiation and growth of sex organs strongly depends on androgens, the reduction in the level of androgen can be a reason for degenerative changes in the tissue structure of seminal vesicle. Nevertheless, few studies have been conducted to examine the effects of organ phosphorus pesticides and the histopathology of seminal vesicle. In a study by Olorunshola et al. (2011), a reduction in epithelial complexity and tissue damage to seminal vesicle was reported in the animals treated with chlorpyrifos compared to the control group (Wissem, 2011). These studies reveal that organ phosphorus pesticides can affect the quality of semen by changing the functioning of seminal vesicles in treated animals. Thus, based on these results, phosalone leads to toxic effects on male fertility.

\section{References:}

1. Adamkovicova M, Toman R, Martiniakova M, Omelka R, Babosova R, et al. (2016), Sperm motility and morphology changes in rats exposed to cadmium and diazinon. Reproductive Biology and Endocrinology. 14: 42.

2. Ambali S, Akanbi, Oladipo L, Yaquband M. (2011), Subchronic chlorpyrifos-induced clinical, haematological and biochemical changes in swiss albino Mice, Protective effect of vitamin E. Int. J. Biol. Med. Res; 2(2): 497-503.

3. Bretveld R, Thomas C, Scheepers P, Zielhuis A, Roeleveld N. (2006), Pesticide exposure: the hormonal function of the female reproductive system disrupted. Reproductive Biology and Endocrinology, 4(30): 4-30. 7.

4. Colborn T, vom Saal FS, Soto AM. (1993). Developmental affects ofendocrinedisrupting chemicals in wildlife and humans. Environ Health Perspect. 101:378-384. 3.

5. Farag A, Eweidah M, El-Okazy A. (2000), Reproductive toxicology of acephate in male mice.Author information.; 5:16-23.

6. Farag A, Radwana A, Sorourb F, Okazyc A, Agamyd E, Sebaea A. (2010), Chlorpyrifos induced reproductive toxicity in male mice.Reproductive Toxicology,29 (1): 80-85.

7. Fattahi E, Parivar S, Moghadamnia A. (2009), The effects of diazinon on testosterone, FSH and LH levels and testicular tissue in mice. Iranian Journal of Reproductive Medicine. 7(2): 59-64.

8. Genga X, Shao H, Zhang Zh, Ng J, Peng Ch. (2015), Malathion-induced testicular toxicity is associated with spermatogenic apoptosis and alterations in testicular enzymes 
and hormone levels in male Wistar rats. Environmental Toxicology and Pharmacology. $1-32$

9. Hanke W, Jurewicz J. (2004). The risk of adverse reproductive and developemental disorders due to occupantional pesticide exposure and overview of current epidemiological evidence. International Journal of Occupational Medicine and Environmental Health, 17(2): 223 - 243.

10. Jasuja N, Sharma P, Suresh C. (2013), A comprehensive effect of acephate on cauda epididymis and accessory sex organs of male rats. African Journal of Pharmacy and Pharmacology. 7(23): 1560-1567.

11. Jorgenson J, Aldrin D (2001), Areview of research on their production, environmental deposition and fate, bioaccumulation, toxicology, and epidemiology in the United States. Environmental health perspectives. 109(Suppl 1):113-9.

12. Joshi S, Mathur R, Gulati N. (2007), Testiculartoxicity of chlorpirifos (an organophosphate pesticide) in albino rat. Toxicology Industrial Health. 23(7): 1525-9.

13. Joshi S, Sharma P. (2011), Male Reproductive Toxicity of Organophosphorous Compounds. Toxicol Environ Chem. 93:1486-1507.

14. kamura A, Kamijim M, Shibata E, OhtaniK, TakagiK. (2015), A comprehensive evaluation of the testicular toxicity of dichlorvos in wistar rats. Toxicology. 213: 129137.

15. Kidd S, Eskenazi B, Wyrobek A. (2001), Effects of male age on semen quality and fertility: a review of the literature. Fertility and sterility. 75(2): 237-48.

16. Kniewald J, Jakominic M, Tomljenovic A, simic B, Romac P, Vranesic D, et al. (2000), Disordersof male rat reproductive tract under theinfluence of atrazine. Journal of AppliedToxicology. 20(1):61-8.

17. Larsen SB, Giwercman A, Spano M, Bonde JP. (1998), A longitudinal study of semen quality in pesticide spraying Danish farmers. The ASCLEPIOS Study Group. Reprod Toxicol; 12:581-589.

18. Melissa J. (2008), Effects of environmental and occupational pesticide exposure on human sperm. Human Reproduction Update. 14(3): 233-242.

19. Najafi Gh, Hobenaghi R, Hoshyari A, Moghadaszadeh, Ghorbanzadeh B. (2013), The effect of atrazine on spermic parameters and fertility potential in mature rats. Arak Medical University Journal. 15(69): 85-94.

20. Nithya R, Elango V. (2015), Pesticide Effect in Male Hormones and Antioxidant Status in Male Albino Rats. Journal of Academia and Industrial Research. 63(4): 2278- 5213

21. Olorunshola K, Achie L. (2011), Ascorbic acid ameliorates toxic effects of chlopyrifos on testicular functions of albino rats. British Journal of Pharmacology and Toxicology. 2(5): 262-269. 
22. Patrick K, Udowelle N, Okereke C. (2016), Testicular toxicity and sperm quality following exposure to Solignum: A Permethrin-containing wood preservative in adult male Wistar rats. Journal of Interdisciplinary Histopathology. 4(1): 13-16.

23. Saenphet K, Jitjaingam A, Chaiyapo M. (2015), Reproductive Toxicity Test of PlantDerived Insecticide in Male Rats. Chiang Mai J. Sci; 42(4): 816-821.

24. Schlegel P, Chang T, Marshall F. (1991), Antibiotics: potential hazards to male fertility.Fertility and Sterility. 55(2): 235-42.

25. Sharma B, Sharma P, Joshi S. (2016), Effect of an organophosphorus insectide diche orovos on the reproductive performance in male rat. European Journal of Biomedical AND Pharmaceutical sciences. 8: 403-409.

26. Souheila S, Mohamed Salah B, Cherif A. (2011), Pesticide exposure and reproductive biomarkers among male farmers from north-east Algeria. Annals of Biological Research, 290-297. 9310.

27. Sujatha R, Chitra K, Latchoumycandane C, Mathur P. (2001), Effect of lindane on testicular antioxidant system and steroidogenic enzymes in adult rats. Asian J Androl; 3: $135-8$.

28. TabbMM, Blumberg B. (2006), New modes of action for endocrine-disrupting chemicals. Mol. Endocrinol., 20: 475-482.

29. Taib I, Budin S, Ghazali A, Jayusman P, Mohamed J. (2014), Fenitrothion alters sperm characteristics in rats: Ameliorating effects of palm oil tocotrienol-rich fraction. Exp. Anim.; 63(4): 383-393.

30. Toppari J, Larsen JC, Christiansen P, Giwercman A, Grandjean P, Guillette LJ, et al. (1996), Male reproductive health and environmental xenoestrogens. Environ Health Perspect, 104 (4):741-803.

31. Wasim A, Dwaipayan S, Ashim C. (2009), Impact of pesticides use in agriculture. Interdisc Toxicol. 2(1): 1-12

32. Whorton M. (1994), Male occupational reproductive hazards. In: ZENZ C. (Editor). Occupational medicine, 3th Edition. Mosby. 2: 870-3. 12.

33. Wissem M, Aziza I, Hadj H, Aicha B, Aghleb B, Olivier T ,et.al. (2011), Effect of Endocrine disruptor pesticides. Int. J. Environ. Res. PublicHealth.; 8: 2265-2303.

34. Wissem M, Aziza I, Hadj H, Aicha B, Aghleb B, Olivier T, Benoit R. (2011), Effect of Endocrine Disruptor Pesticides. Int. J. Environ. Res. Public Health, 8: 2265- 2303. 
